\title{
Mechanism of Oxidative Shuttling for [2]Rotaxane in Stoddart-Heath Molecular Switch: Density Functional Theory Study with Continuum-Solvation Model
}

\author{
Yun Hee Jang ${ }^{\dagger}$ and William A. Goddard, III* \\ Materials and Process Simulation Center, Beckman Institute (139-74) \\ California Institute of Technology, Pasadena, California 91125
}

\section{Supporting Information}

Table S1. Ionization potentials (IP) and reduction potentials $\left(E^{0}\right)$ of TTF and DNP.

\begin{tabular}{|c|c|c|c|c|c|c|c|c|c|c|}
\hline & DFT & Opt & $E(\mathrm{~g})$ & $\mathrm{ZPE} / \Delta \Delta G_{0 \rightarrow 298 \mathrm{~K}}$ & Opt(sol) & $\Delta G_{\text {solv }}$ & $E^{0}(\mathrm{TTF})$ & $E^{0}(\mathrm{DNP})$ & $\mathrm{IP}^{\mathrm{a}}(\mathrm{TTF})$ & $\mathrm{IP}^{\mathrm{a}}(\mathrm{DNP})$ \\
\hline (a) & B3LYP & $6-31 G^{* *}$ & 6-31G** & - & - & 6-31G** S $1.9 \AA$ & $0.22 / 1.05$ & 0.88 & $6.1^{b}(6.3)^{c}$ & $6.6^{\mathrm{b}}(6.7)^{\mathrm{c}}$ \\
\hline (b) & B3LYP & $6-31 \mathrm{G}^{* *}$ & $6-31 G^{* *}$ & - & $6-31 G^{* *}$ & $6-31 G^{* *}$ S $1.9 \AA$ & $0.22 / 1.04$ & 0.89 & & \\
\hline (c) & B3LYP & $6-31 \mathrm{G}^{* *}$ & $6-31 G^{* *}$ & $6-31 G^{* *}$ & $6-31 G^{* *}$ & 6-31G** S $1.9 \AA$ & $0.29 / 1.07$ & 0.89 & & \\
\hline (d) & B3LYP & $6-31 G^{* *}$ & $6-311++G^{* *}$ & - & - & $6-31 G^{* *} \mathrm{~S} 1.9 \AA$ & $0.41 / 1.12$ & 1.17 & $6.3(6.5)$ & $6.8(7.1)$ \\
\hline (e) & B3LYP & $6-31 G^{* *}$ & $6-311++G^{* *}$ & - & $6-31 G^{* *}$ & $6-31 G^{* *}$ S $1.9 \AA$ & $0.41 / 1.11$ & 1.18 & & \\
\hline (f) & B3LYP & $6-31 G^{* *}$ & $6-311++\mathrm{G}^{* *}$ & $6-31 G^{* *}$ & $6-31 G^{* *}$ & 6-31G** S $1.9 \AA$ & $0.48 / 1.14$ & 1.18 & & \\
\hline (g) & B3LYP & $6-31 \mathrm{G}^{* *}$ & cc-pVTZ(-f)++ & - & - & $6-31 G^{* *}$ S $1.9 \AA$ & $0.38 / 1.07$ & & (6.5) & \\
\hline (h) & B3LYP & $6-31 G^{* *}$ & cc-pVTZ(-f)++ & $6-31 G^{* *}$ & - & $6-31 G^{* *} \mathrm{~S} 1.9 \AA$ & $0.44 / 1.10$ & & & \\
\hline (i) & B3LYP & $6-31 G^{* *}$ & cc-pVTZ(-f)++ & $6-31 G^{* *}$ & $6-31 G^{* *}$ & $6-31 G^{* *}$ S $1.9 \AA$ & $0.45 / 1.09$ & & & \\
\hline (d') & B3LYP & $6-31 G^{* *}$ & $6-311++\mathrm{G}^{* *}$ & - & - & $6-31 \mathrm{G}^{* *} \mathrm{~S} 1.7 \AA$ & $0.32 / 0.90$ & 1.18 & $6.3(6.5)$ & $6.8(7.1)$ \\
\hline & Expt'l & & & & & & $\begin{array}{l}0.3-0.4 / \\
0.7-0.8^{d}\end{array}$ & $1.1-1.3^{\mathrm{e}}$ & $6.3(6.7)^{\mathrm{f}}$ & $(7.4)^{\mathrm{e}}$ \\
\hline
\end{tabular}

${ }^{\mathrm{a}}$ From the energy difference between neutral and cationic species. ${ }^{\mathrm{b}}$ Vertical IP. ${ }^{\mathrm{c}}$ Adiabatic IP. ${ }^{\mathrm{d}} \operatorname{Ref}{ }^{1-3}$.

${ }^{\mathrm{e}}$ 1,5-dimethoxynaphthalene, $\operatorname{Ref}^{4}$. ${ }^{\mathrm{f}} \operatorname{Ref}^{3,5}$. ${ }^{\mathrm{g}}$ Estimated (7.38) and measured (7.41) for 1,5-dimethoxynaphthalene. Ref ${ }^{6}$.

Table S2. Master table.

\begin{tabular}{|c|c|c|c|c|c|c|c|c|c|c|}
\hline & $S$ & $\Delta E$ & $\Delta G_{\text {solv }}$ & $\Delta G_{A N}^{0}$ & q(TTF) & q(DNP) & s(TTF) & $\mathrm{s}(\mathrm{DNP})$ & $\Delta \Delta G_{A N}^{0}$ & $E^{0}$ \\
\hline${ }^{1}$ 2: TTF & 0 & -1144514.8 & -5.6 & -1144520.5 & $0.00 / 0.00$ & - & - & - & - & - \\
\hline${ }^{2} \mathbf{2}^{+}: \mathrm{TTF}^{+}$ & $1 / 2$ & -1144368.6 & -49.4 & -1144418.0 & $1.00 / 1.00$ & - & 1.0 & - & - & 0.32 \\
\hline${ }^{1} \mathbf{2}^{2+}: \mathrm{TTF}^{2+}$ & 0 & -1144115.0 & -187.2 & -1144302.2 & $2.00 / 2.00$ & - & - & - & 0.0 & 0.90 \\
\hline${ }^{3} \mathbf{2}^{2+}: \mathrm{TTF}^{2+}$ & 1 & -1144078.3 & -185.4 & -1144263.7 & $2.00 / 2.00$ & - & 2.0 & - & 38.5 & - \\
\hline${ }^{1}$ 3: DNP & 0 & -435340.9 & -6.5 & -435347.3 & - & $0.00 / 0.00$ & - & - & - & - \\
\hline${ }^{2} \mathbf{3}^{+}: \mathrm{DNP}^{+}$ & $1 / 2$ & -435183.0 & -42.2 & -435225.2 & - & $1.00 / 1.00$ & - & 1.0 & - & 1.18 \\
\hline${ }^{14}$ 4: C(TTF)P & 0 & -4516518.5 & -74.2 & -4516592.7 & $0.16 / 0.32$ & - & - & - & - & - \\
\hline${ }^{2} \mathbf{4}^{+}: \mathrm{C}(\mathrm{TTF}) \mathrm{P}^{+}$ & $1 / 2$ & -4516361.5 & -117.0 & -4516478.5 & $0.72 / 0.70$ & - & 0.94 & - & - & 0.83 \\
\hline${ }^{1} \mathbf{4}^{2+}: \mathrm{C}(\mathrm{TTF}) \mathrm{P}^{2+}$ & 0 & -4516119.6 & -207.7 & -4516327.3 & $1.52 / 1.28$ & - & - & - & 0.0 & 2.44 \\
\hline${ }^{3} \mathbf{4}^{2+}: \mathrm{C}(\mathrm{TTF}) \mathrm{P}^{2+}$ & 1 & -4516094.3 & -208.5 & -4516302.7 & $1.16 / 0.99$ & - & 1.49 & - & 24.5 & - \\
\hline${ }^{1} 5: \mathrm{C}(\mathrm{DNP}) \mathrm{P}$ & 0 & -3807336.3 & -71.9 & -3807408.2 & - & $-0.09 /-0.06$ & - & - & - & - \\
\hline${ }^{2} 5^{+}: C(D N P) P^{+}$ & $1 / 2$ & -3807167.3 & -111.5 & -3807278.7 & - & $0.73 / 0.55$ & - & 0.98 & - & 1.49 \\
\hline${ }^{1} \mathbf{6 b}:$ TTF-DNP & 0 & -1579098.9 & -11.3 & -1579110.2 & $-0.08 /-0.15$ & $0.08 / 0.15$ & - & - & - & - \\
\hline${ }^{2} \mathbf{6 b}^{+}:$TTF-DNP ${ }^{+}$ & $1 / 2$ & -1578957.8 & -48.1 & -1579005.9 & $0.87 / 0.84$ & $0.13 / 0.16$ & 1.00 & 0.00 & - & 0.40 \\
\hline${ }^{1} \mathbf{6 b}^{2+}:$ TTF-DNP ${ }^{2+}$ & 0 & -1578744.2 & -140.1 & -1578884.3 & $1.81 / 1.80$ & $0.19 / 0.20$ & - & - & 0.0 & 1.15 \\
\hline${ }^{3} \mathbf{6 b}^{2+}:$ TTF-DNP ${ }^{2+}$ & 1 & -1578756.8 & -126.4 & -1578883.2 & $0.89 / 0.84$ & $1.11 / 1.16$ & 1.00 & 1.00 & 1.1 & - \\
\hline${ }^{2} \mathbf{6 b}^{3+}:$ TTF-DNP ${ }^{3+}$ & $1 / 2$ & -1578475.3 & -289.2 & -1578764.5 & $1.84 / 1.82$ & $1.16 / 1.18$ & 0.00 & 1.00 & - & 1.07 \\
\hline${ }^{1}$ 7: C(TTF)P-DNP & 0 & -4951104.1 & -72.2 & -4951176.3 & $0.12 / 0.28$ & $0.01 / 0.02$ & - & - & - & - \\
\hline${ }^{2} \mathbf{7}^{+}: \mathrm{C}(\mathrm{TTF}) \mathrm{P}-\mathrm{DNP}^{+}$ & $1 / 2$ & -4950955.3 & -106.9 & -4951062.3 & $0.61 / 0.60$ & $0.09 / 0.07$ & 0.93 & 0.02 & - & 0.83 \\
\hline${ }^{1} \mathbf{7}^{2+}:$ C(TTF)P-DNP ${ }^{2+}$ & 0 & -4950744.0 & -177.8 & -4950921.9 & $0.85 / 0.75$ & $0.79 / 0.78$ & - & - & 10.7 & - \\
\hline${ }^{3} \mathbf{7}^{2+}: \mathrm{C}(\mathrm{TTF}) \mathrm{P}-\mathrm{DNP}^{2+}$ & 1 & -4950753.7 & -178.8 & -4950932.5 & $0.63 / 0.59$ & $1.05 / 1.05$ & 0.95 & 1.00 & 0.0 & 1.50 \\
\hline 8: TTF-C(DNP)P & 0 & -4951093.0 & -75.5 & -4951168.4 & $-0.06 /-0.18$ & $-0.04 / 0.12$ & - & - & - & - \\
\hline${ }^{2} \mathbf{8}^{+}:$TTF-C(DNP) $\mathrm{P}^{+}$ & $1 / 2$ & -4950944.3 & -113.2 & -4951057.5 & $0.87 / 0.75$ & $0.00 / 0.17$ & 0.98 & 0.02 & - & $1.03(0.69)^{\mathrm{a}}$ \\
\hline${ }^{1} \mathbf{8}^{2+}:$ TTF-C(DNP)P ${ }^{2+}$ & 0 & -4950724.0 & -200.3 & -4950924.3 & $1.49 / 1.36$ & $0.31 / 0.40$ & - & - & 2.1 & - \\
\hline${ }^{3} \mathbf{8}^{2+}:$ TTF-C(DNP)P ${ }^{2+}$ & 1 & -4950736.5 & -189.8 & -4950926.4 & $0.93 / 0.79$ & $0.79 / 0.75$ & 1.00 & 0.98 & 0.0 & $1.77(1.56)^{\mathrm{a}}$ \\
\hline
\end{tabular}

${ }^{1}$ 9: C(TTF)P:DNP $\quad 0 \quad-4951862.3 \quad-68.3 \quad-4951930.6$

${ }^{a}$ Reduction potential with respect to the metastable [TTF-C(DNP)P] in parenthesis.

\section{References}

(1) Ashton, P. R.; Baldoni, V.; Balzani, V.; Claessens, C. G.; Credi, A.; Hoffmann, H. D. A.; Raymo, F. M.; Stoddart, J. F.; Venturi, M.; White, A. J. P.; Williams, D. J. Eur. J. Org. Chem. 2000, 1121-1130.

(2) Balzani, V.; Credi, A.; Mattersteig, G.; Matthews, O. A.; Raymo, F. M.; Stoddart, J. F.; Venturi, M.; White, A. J. P.; Williams, D. J. J. Org. Chem. 2000, 65, 1924-1936.

(3) Lichtenberger, D. L.; Johnston, R. L.; Hinkelmann, K.; Suzuki, T.; Wudl, F. J. Am. Chem. Soc. 1990, 112.

(4) Zweig, A.; Maurer, A. H.; Roberts, B. G. J. Org. Chem. 1967, 32, 1322-1329.

(5) Saito, G.; Enoki, T.; Mototada, K.; Imaeda, K.; Sato, N.; Inokuchi, H. Mol. Cryst. Liq. Cryst. 1985, 119, 393-400.

(6) El-Kemary, M. Can. J. Appl. Spectrosc. 1996, 41, 109-113. 